\title{
CARACTERIZAÇÃO HIDROGEOQUÍMICA DE CABECEIRAS DE DRENAGEM NA SERRA DA MANTIQUEIRA
}

\author{
José Oliveira de Almeida Neto $^{(\mathrm{a})}$, Miguel Fernandes Felippe ${ }^{(\mathrm{b})}$, Antônio Pereira Magalhães \\ Júnior $^{(\mathrm{c})}$, Fernando César da Costa ${ }^{(\mathrm{d})}$
}

(a) Curso de Geografia/Instituto de Ciências Humanas, Universidade Federal de Juiz de Fora, jose.oliveira95@hotmail.com.br

(b) Departamento de Geociências/Instituto de Ciências Humanas, Universidade Federal de Juiz de Fora, miguel.felippe@ufjf.edu.br

(c) Departamento de Geografia/Instituto de Geociências, Universidade Federal de Minas Gerais, magalhaesufmg@yahoo.com.br

(d) Departamento de Geografia/Instituto de Geociências, Universidade Federal de Minas Gerais, labgeomorfo@igc.ufmg.br

\section{EIXO: SISTEMAS GEOMORFOLÓGICOS: ESTRUTURA, DINÂMICAS E PROCESSOS.}

\section{Resumo}

O objetivo deste trabalho é avaliar as características hidrogeoquímicas de três cabeceiras de drenagem na Serra da Mantiqueira. Foram estudadas três bacias de cabeceira na Reserva Biológica Poço D’Anta, no município de Juiz de Fora-MG, na borda noroeste da Serra da Mantiqueira Meridional. A metodologia baseou-se em análises laboratoriais de características físico-químicas de amostras de águas coletadas em pontos de diferentes hierarquias fluviais nas três cabeceiras. Os resultados evidenciam que, apesar de concentrações similares dos parâmetros mensurados (resultado da uniformidade hidrogeológica), há nuances referentes à hierarquia fluvial e às características hidrogeomorfológicas, pedológicas e biológicas do ambiente.

Palavras chave: cabeceira; carga em solução; evolução do relevo.

\section{Introdução}

As cabeceiras de drenagem são complexos sistemas (hidro)geomorfológicos que engendram processos fluviais, subsuperficiais e de encosta, em uma área diminuta, marcada por nascentes, proto-canais e canais temporários e perenes que fazem a conexão com vales fluviais de maior hierarquia. Na concepção de Strahler (1957), as bacias de cabeceiras (headwater) restringir-se-iam à superfície drenada por canais de primeira e segunda ordens. Nesse sentido, abrangem 60 a $80 \%$ de todo o acumulado da extensão da drenagem fluvial (SCHUMM, 1956).

Notadamente, a morfologia das cabeceiras traduz uma interação entre "solos residuais, depósitos de movimentos de massa, madeira, blocos, finas camadas de alúvios mal selecionados e afloramentos da rocha matriz" (BENDA et al., 2005). Sua evolução geomorfológica, sobretudo em clima tropical, é associada a movimentos de massa e voçorocamentos, continuados por processos erosivos lineares (SHUMM, 1956; MONTGOMERY e DIETRICH, 1988; MONTGOMERY e DIETRICH, 1989), promovendo sua forma majoritariamente côncava e alinhada pela rede de drenagem. Os trabalhos de 
Dunne (1980), entretanto, indicaram a importância de se observar os processos subsuperficiais para a evolução da rede de drenagem e, consequentemente, das cabeceiras.

Dada a sua função enquanto zonas de descarga, as cabeceiras de drenagem promovem substanciais interações entre as águas subsuperficiais, superficiais e meteóricas, formando turfeiras, surgências e nascentes (PALMER et al., 2001; HAIGH, 2006; LEVISON et al., 2013; FELIPPE e MAGALHÃES JR, 2014; FELIPPE e MAGALHÃES JR, 2016). Nesse sentido, não se pode negar a importância dos processos subsuperficiais na evolução gemorfológica de cabeceiras (DUNNE, 1980; FELIPPE, 2013), sobretudo aqueles relacionados à desnudação geoquímica quando em ambiente tropical úmido.

As características químicas das águas possuem profunda relação com o material inorgânico e orgânico com o qual entrou em contato, traduzindo sua história. A água meteórica, já enriquecida por gases e particulados da atmosfera, entra em contato com a superfície carreando solutos em direção ao meio subterrâneo durante a percolação. Em contato com o aquífero, os processos de intemperismo químico atuam liberando na água compostos e íons lixiviados. Todo esse material aflora novamente à superfície por meio da exfiltração (DAVIS, 1966; FEITOSA e MANOEL-FILHO, 2000; ALBAREDE, 2011). Em cabeceiras de drenagem, esse processo ganha importância, devido à concentração de nascentes e surgências, retirando massa do meio subterrâneo e contribuindo para o esvaziamento geoquímico da bacia hidrográfica (FELIPPE e MAGALHÃES JR, 2016).

Apesar de sua grande importância para a dinâmica fluvial e ambiental, as cabeceiras de drenagem ainda são pouco compreendidas. Existem grandes lacunas no entendimento da interação entre processos atmosféricos, hidrológicos e geomorfológicos, no papel que possuem para as bacias hidrográficas, nas relações ecológicas em seus distintos ambientes, na relação dos aquíferos com os canais fluviais e, não menos importante, na desnudação geoquímica (GOMI et al., 2002; LEVISON et al., 2013; BORGA et al., 2014; FELIPPE e MAGALHÃES JR, 2016).

Nesse ínterim, o objetivo deste trabalho é o de avaliar as características hidrogeoquímicas de três cabeceiras de drenagem na Serra da Mantiqueira. Estes esforços somam-se a outros de natureza similar abrangidos pelo projeto "O papel das nascentes e cabeceiras de drenagem na evolução de unidades do relevo de Minas Gerais por meio da análise dos processos de desnudação geoquímica" (CNPq 441353/20142).

Foram estudadas três bacias de cabeceira ( $2^{\mathrm{a}}$ ordem de Strahler $3^{\mathrm{a}}$ magnitude de Shreve) na Reserva Biológica Poço D’Anta, no município de Juiz de Fora-MG, na borda noroeste da Serra da Mantiqueira Meridional. A partir de análises laboratoriais de amostras de águas coletadas, mensurou-se os sólidos totais dissolvidos, a condutividade elétrica, a alcalinidade e a concentração de sílica de nascentes e cursos d'água. 
XVII Simpósio Brasileiro

de Geografia Física Aplicada

I Congresso Nacional

de Geografia Física
OS DESAFIOS DA GEOGRAFIA FÍSICA NA FRONTEIRA DO CONHECIMENTO

Instituto de Geociências - Unicamp

Campinas - SP

28 de Junho à 02 de Julho de 2017

\section{Caracterização da área de estudo}

A Reserva Biológica Municipal Poço D’Anta está inserida no perímetro urbano do município de Juiz de Fora/MG (Figura 1). Ocupando cerca de 277 ha, a reserva é um fragmento em estágio avançado de regeneração de Mata Atlântica (Formação Florestal Estacional Semidecidual) que, na segunda metade do século XIX, sofreu grandes perdas de área para a implantação do ciclo do café. Além dos fragmentos de mata nativa, que vem sofrendo o processo de regeneração por sucessão secundária, a reserva ainda conta com a presença de espécies exóticas e trechos com Floresta Paludosa (Mata de Brejo) ao longo de canais fluviais (SANTIAGO, RESENDE e FONSECA, 2007; FONSECA, 2012; CRUZ, 2016).

A bacia de estudo tem seus fluxos de energia controlados pelo nível de base local, uma lagoa artificial, por depósitos de blocos e por fragmentos de vegetação que atuam em canais de primeira ordem como soleiras geomorfológicas. Tais soleiras condicionam a presença de Floresta Paludosa, favorecem a reprodução da fauna, e por vezes, tornam a drenagem descontinua. O ladrão da lagoa drena para o Córrego das Antas, afluente da margem esquerda do rio Paraibuna (IBGE, 1976; FARIA, 1996).

Oliveira et al. (2014), ao analisarem as variáveis climatológicas radiação e precipitação, apontam que a área de estudo apresenta uma sazonalidade bem definida, composta por duas estações bem diferenciadas: a estação mais chuvosa, mais irradiada e quente vai de outubro a março; enquanto a estação mais seca, menos irradiada e fria vai de abril a setembro.

O município está localizado na unidade geomorfológica Serranias da Zona da Mata, região da Mantiqueira Meridional. A Mantiqueira é caracterizada por um conjunto de cristas montanhosas, parcamente alinhadas no sentido SSW-NNE, que se estende de São Paulo até o norte do Espírito Santo. Apresenta-se como uma das porções mais elevadas do Brasil oriental, resultante de uma complexa história geológica que remonta à separação da placa afro-brasileira, abarcando soerguimentos paleozoicos, rifteamento mesozoico e soerguimentos diferenciais cenozoicos (SAADI, 1991; MARQUES-NETO et al., 2015). Compreendida pelo denominado Rift Continental do Sudeste do Brasil, por Riccomini (1989), o conjunto serrano da Mantiqueira configura-se em um horst associado ao gráben do Paraíba do Sul cronocorrelato e geneticamente ligado ao horst da Serra do Mar.

O município de Juiz de Fora localiza-se no extremo norte do compartimento da Mantiqueira Meridional, no contato com o Planalto do Campo das Vertentes (IBGE, 2006). Seu relevo é fortemente influenciado por estruturas geológicas pretéritas com indícios de reativação neotectônica, promovendo a ocorrência de uma depressão intramontana que abriga o rio Paraibuna (principal curso d'água do município) circundada por feições de morros e morrotes esculpidos por dissecação fluvial sobre materiais elúvio-coluviais deslocados em direção à depressão. Nesse sentido, as cabeceiras tendem a ser côncavas e de declividade suave, abrigando nascentes que ocorrem em aquíferos suspensos no material inconsolidado ou no contato 


\section{OS DESAFIOS DA GEOGRAFIA FÍSICA NA FRONTEIRA DO CONHECIMENTO \\ Instituto de Geociências - Unicamp \\ Campinas - SP \\ 28 de Junho à 02 de Julho de 2017}

rocha/solo em locais de mantos de alteração mais rasos. As cabeceiras de estudo são apresentadas nas Figuras 2, 3 e 4.

A área de estudo é embasada pelo Complexo Juiz de Fora, composto por Ortogranulito Enderbítico a Charnockítico. Os dados isotópicos de Sr e Nd indicam que a cristalização magmática ocorreu entre 2134 e 2084 Ma. (OLIVEIRA, A., 2016; ROCHA, 2005; NOCE et al, 2007).
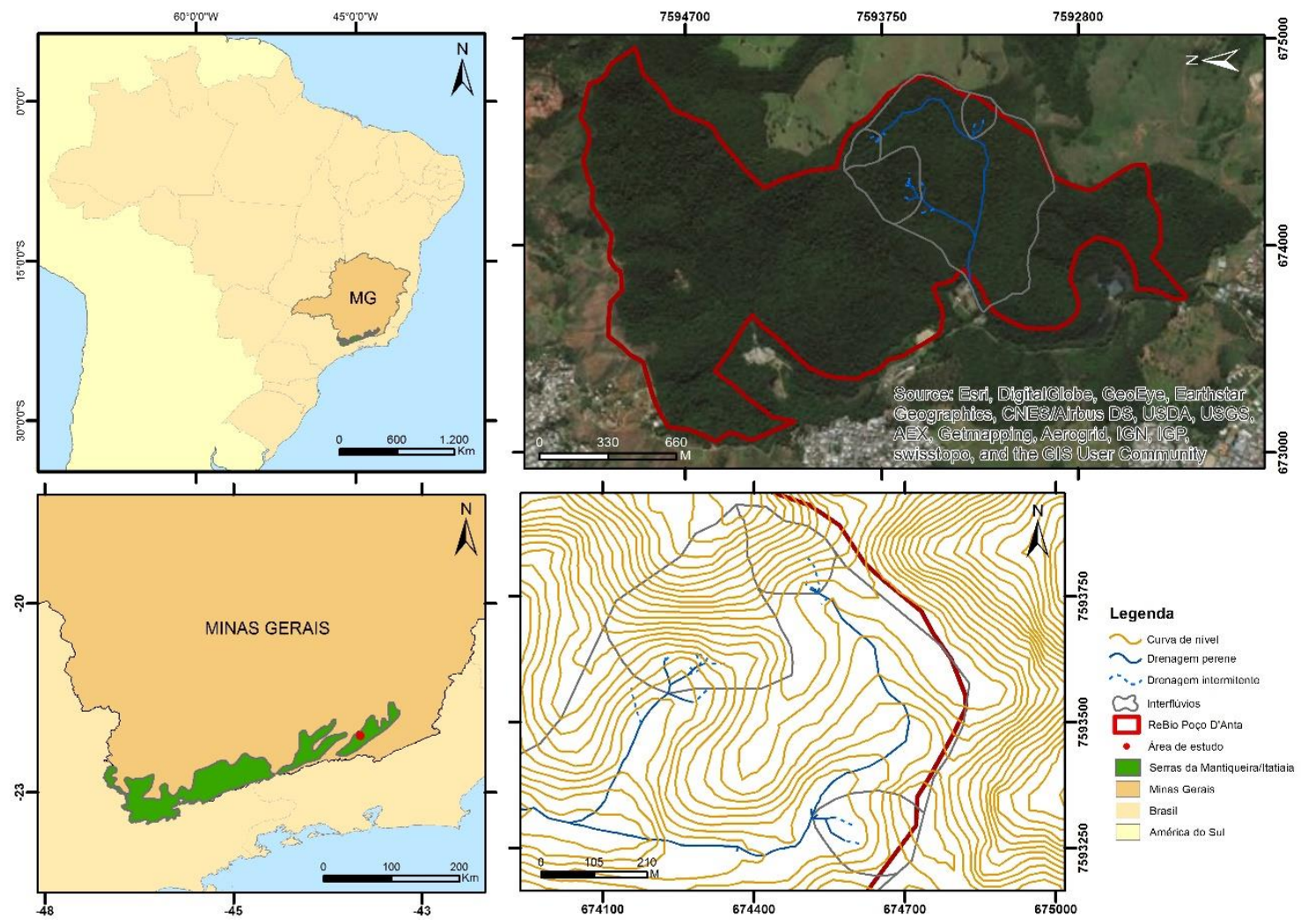

Figura 1 - Localização da área de estudo. 


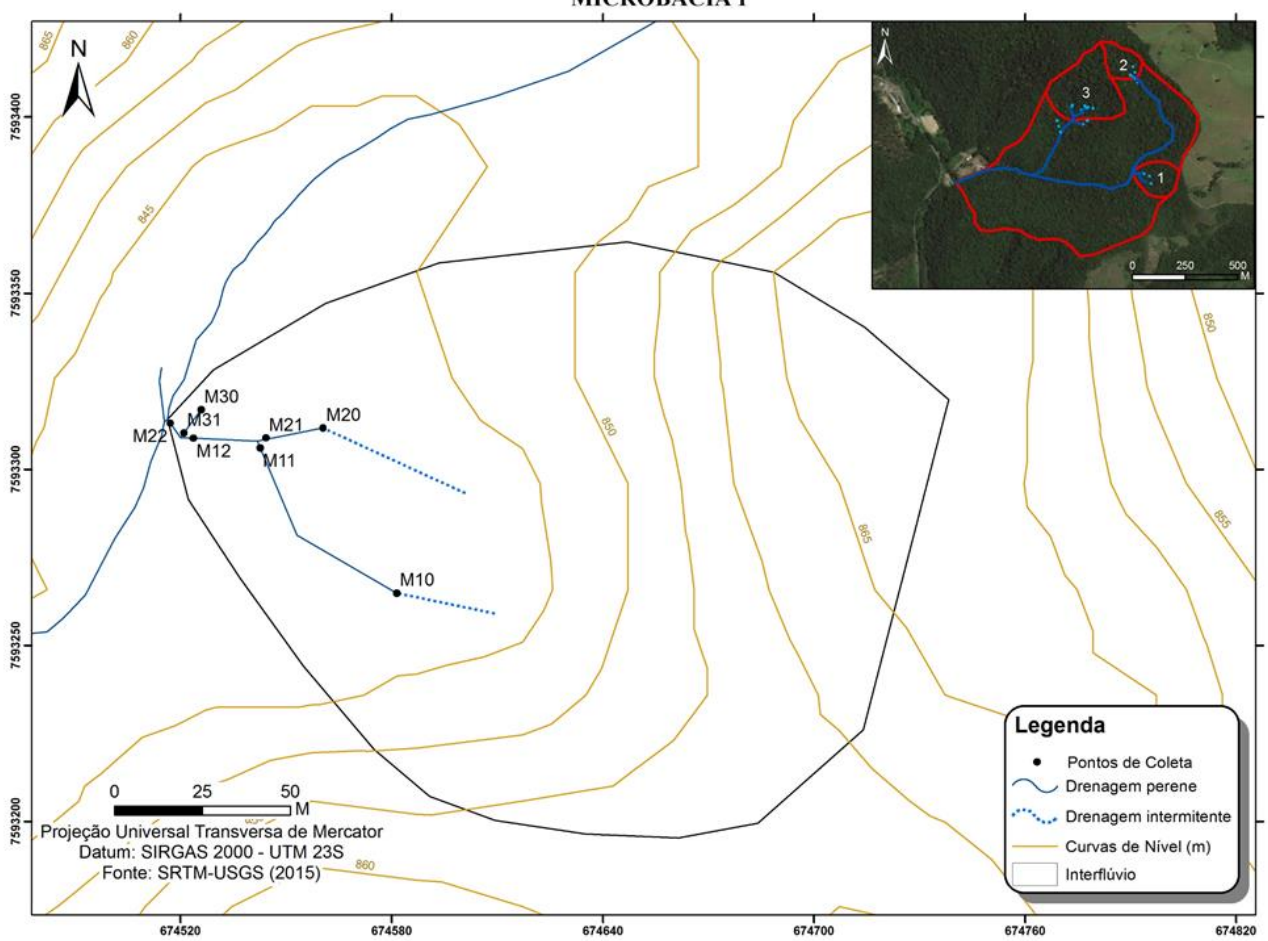

Figura 2 - Microbacia 1.

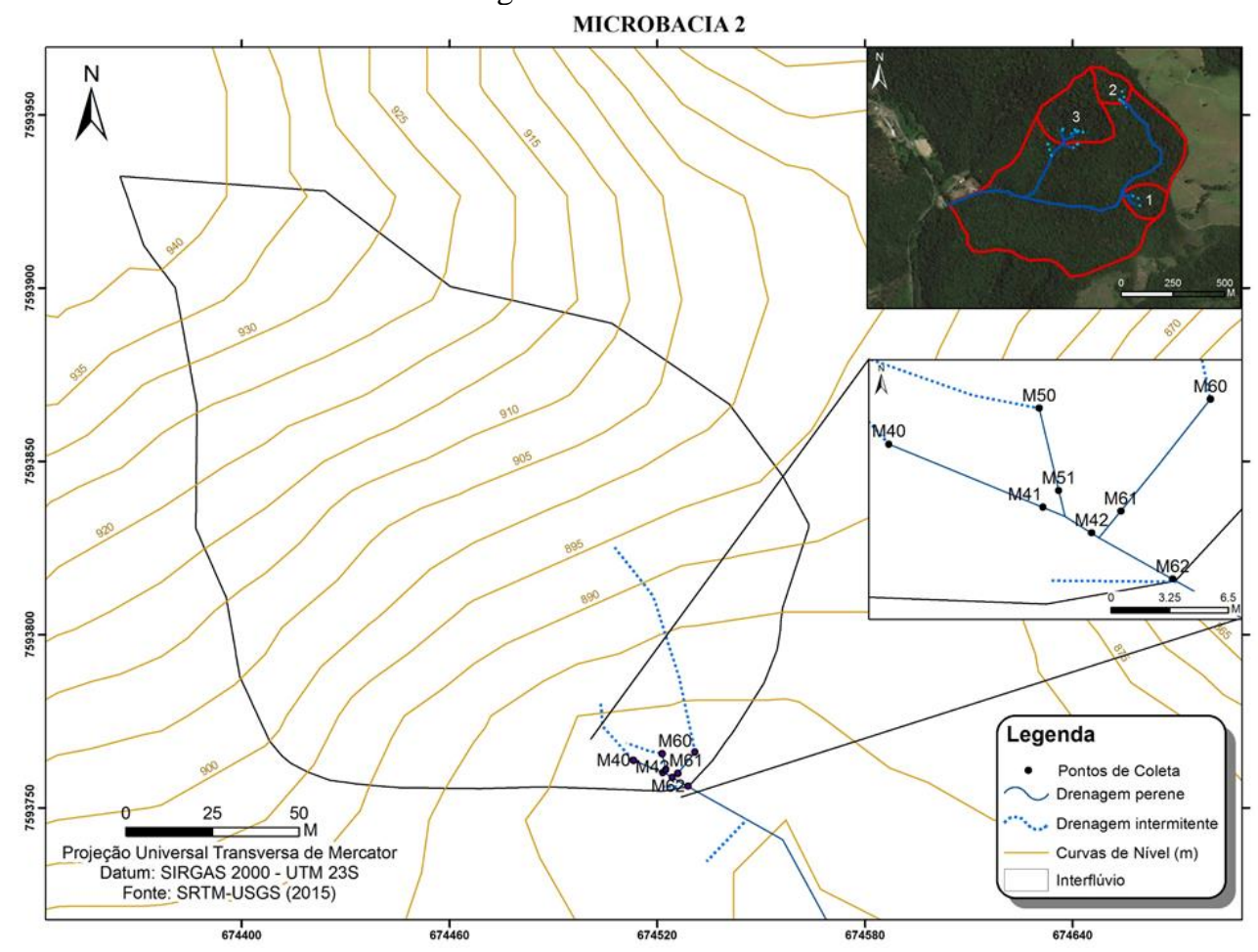

Figura 3 - Microbacia 2. 

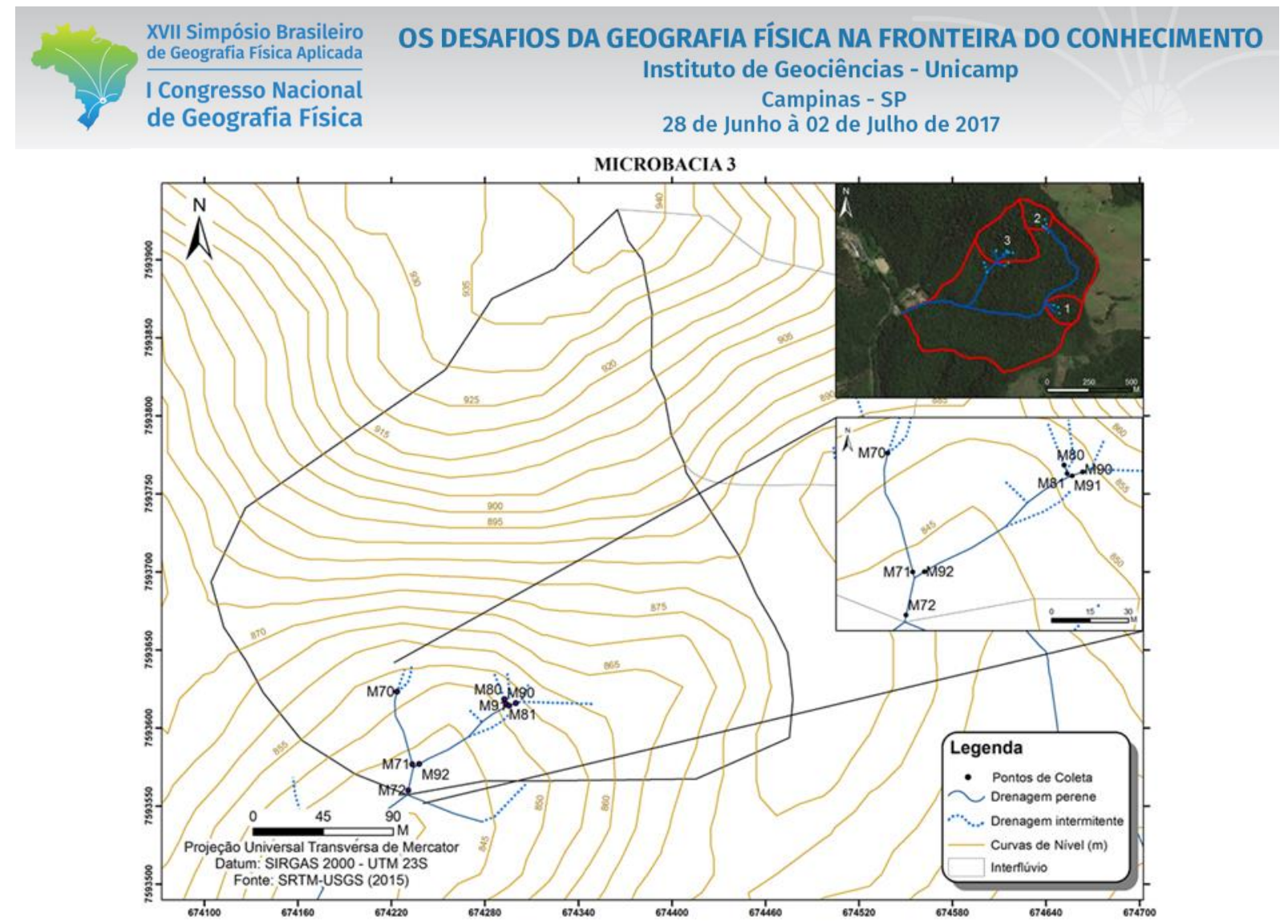

Figura 4 - Microbacia 3.

\section{Metodologia}

As coletas das amostras foram realizadas trimestralmente entre novembro de 2015 a setembro de 2016. Alíquotas de $500 \mathrm{~mL}$ foram acondicionadas em frascos de polipropileno, com a utilização de seringas e enviadas sob refrigeração para análise no Laboratório de Geomorfologia, do Instituto de Geociências, da Universidade Federal de Minas Gerais (UFMG).

A vazão foi calculada através da metodologia preconizada por Felipe e Magalhães Jr. (2009), onde o fluxo de água é coletado em um tempo cronometrado com um saco plástico maleável, tendo seu volume aferido com o uso de seringa com intervalo de graduação de $1 \mathrm{ml}$. Tal operação foi realizada em triplicata a fim de diminuir possíveis erros. A vazão é calculada através da Fórmula 1.

$Q=v o l / t$

(Equação 1)

Onde: $\mathrm{Q}$ - vazão em litros por segundo; $\mathrm{t}$ - tempo de coleta em segundos; vol - volume em litros.

Os acumulados diários de precipitação foram cedidos pelo Laboratório de Climatologia e Análise Ambiental (LabCAA), do Instituto de Ciências Humanas, da Universidade Federal de Juiz de Fora (UFJF). A concentração de Sólidos Totais Dissolvidos (TDS) foi determinada por meio da gravimetria 


\section{OS DESAFIOS DA GEOGRAFIA FÍSICA NA FRONTEIRA DO CONHECIMENTO \\ Instituto de Geociências - Unicamp \\ Campinas - SP \\ 28 de Junho à 02 de Julho de 2017}

medindo-se a variação de massa em balança analítica $(0,0001 \mathrm{~g})$ em uma alíquota de $50 \mathrm{~mL}$ de amostra, antes e depois de secagem completa em estufa a $180^{\circ} \mathrm{C}$. A condutividade elétrica (EC) foi mensurada em laboratório nas amostras in natura em condutivímetro da marca Tecnopon modelo mCa-150, com célula de condutividade $\mathrm{k}=0,1$ calibrado com solução padrão de $146,9 \mu \mathrm{S} / \mathrm{cm}$. A concentração de sílica na água foi determinada por espectrofotometria a partir de amostras filtradas $(0,45 \mu \mathrm{m})$ em sistema de filtração sob pressão reduzida. A reação com molibdato de amônio em pH aproximadamente 1,2 leva formação de ácido molibdossilícico, de coloração amarela, e a intensidade da cor, é proporcional à concentração de sílica contida na amostra (APHA; AWWA; WEF, 2012). O equipamento utilizado na leitura foi o espectrofotômetro da marca Tecnal modelo V500. Foi construída uma curva de calibração $\left(\mathrm{R}^{2}=0,9996\right)$ utilizando soluções de $\mathrm{SiO}_{2}$ com concentrações conhecidas de 2, 5, 10, 20 e $30 \mathrm{mg} / \mathrm{L}$. A alcalinidade das amostras filtradas foi mensurada utilizando titulador potenciométrico da marca HANNA modelo HI902, com titulante $\mathrm{HCl}$ 0,1 $\mathrm{N}$ padronizado. Todos os procedimentos analíticos foram realizados no Laboratório de Geomorfologia da Universidade Federal de Minas Gerais, seguindo os preceitos definidos por APHA (1985).

\section{Resultados e discussão}

Mesmo estando sob o mesmo regime de precipitação (Figura 5), os pontos monitorados apresentaram respostas hidrogeomorfológicas distintas aos eventos meteorológicos. Nota-se uma clara tendência à dupla estacionalidade climática, com aproximadamente $80 \%$ da precipitação anual concentrada nos meses de novembro a março. Com isso, os momentos de monitoramento coincidem com o início do período chuvoso (novembro), o final do mesmo (março), o auge do período seco (junho) e o final do período seco, já em transição para o período úmido (setembro).

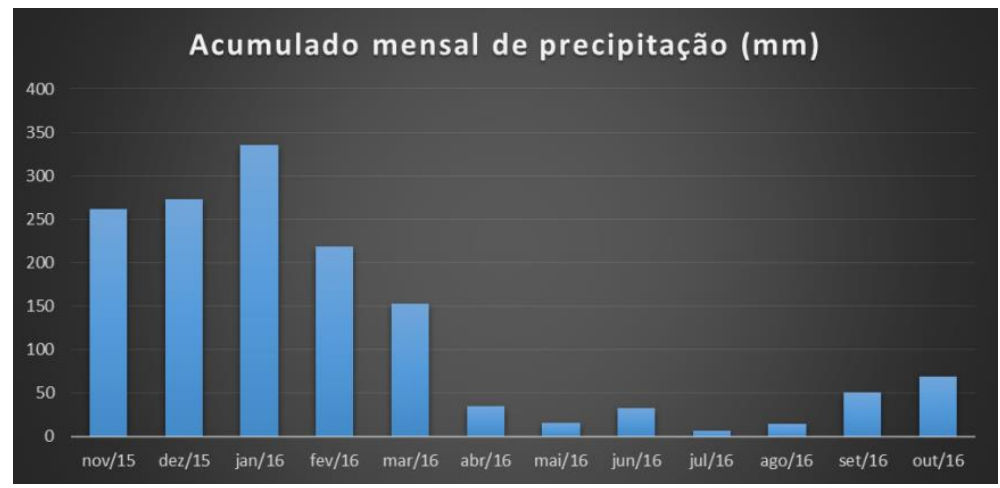

Figura 5 - Acumulados mensais de precipitação.

$\mathrm{Na}$ Tabela 1 são apresentados os valores calculados de vazão para todos os pontos ao longo do ano hidrológico. O aumento geral de vazão de novembro para março é esperado devido à concentração dos 


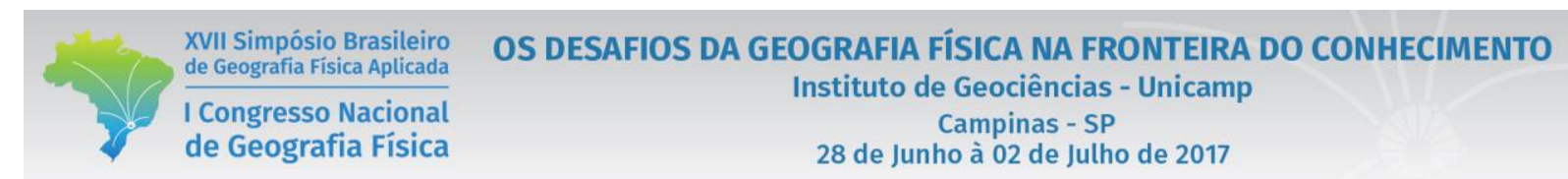

eventos de precipitação no período. Entre os campos de março e junho, o acumulado de precipitação diminui, o que promove diminuição da vazão em grande parte dos pontos. Os expressivos eventos de precipitação do mês de setembro aconteceram após o dia sete, data na qual foram feitas as coletas em campo, fazendo com que os valores de vazão desse mês fossem ainda menores do que nos meses anteriores (mínimo hidrológico). É importante ressaltar que os pontos M72 e M92 não apresentam a série de dados completa por apresentarem, por vezes, vazão superior a detectável pelo método.

Tabela 1 - variação temporal da vazão (L/s).

\begin{tabular}{c|ccccc} 
Micro-bacia & Ponto/Mês & Nov & Mar & Jun & Set \\
\hline \multirow{4}{*}{1} & M10 & 0,007 & 0,005 & 0,045 & 0,026 \\
& M11 & 0,011 & 0,126 & NM & NM \\
& M12 & 0,016 & 0,500 & 0,068 & 0,012 \\
& M20 & S & 0,043 & 0,025 & NM \\
& M21 & S & 0,061 & NM & S \\
& M30 & 0,024 & 0,250 & NM & NM \\
& M31 & NM & 0,004 & NM & S \\
& M40 & S & 0,004 & NM & S \\
\hline \multirow{5}{*}{2} & M41 & S & 0,013 & 0,001 & S \\
& M42 & S & 0,029 & 0,006 & S \\
& M50 & S & 0,003 & 0,019 & S \\
& M51 & S & 0,060 & 0,011 & S \\
& M60 & S & 0,005 & NM & S \\
& M61 & S & 0,011 & S & S \\
& M62 & 0,009 & 0,012 & 0,004 & S \\
\hline \multirow{5}{*}{3} & M70 & NM & 0,074 & 0,039 & 0,008 \\
& M71 & 0,020 & 0,233 & 0,064 & 0,009 \\
& M72 & NM & NM & NM & NM \\
& M80 & 0,005 & 0,028 & 0,023 & 0,034 \\
& M81 & 0,046 & 0,089 & 0,085 & 0,044 \\
& M90 & 0,044 & 0,018 & 0,118 & 0,047 \\
& M91 & 0,062 & 0,174 & NM & NM \\
& M92 & 0,122 & 0,354 & NM & NM
\end{tabular}

Fonte: Dados primários. Legenda: NM - Não mensurável; S - Seca.

Os pontos M10, M50 e M90 (todos referentes a nascentes) apresentaram uma dinâmica peculiar. Enquanto a maioria dos pontos teve um aumento de sua vazão de novembro para março, os pontos M10 e M90 tiveram um declínio nos valores absolutos de vazão, seguidos por um aumento no mês de junho e um novo declínio no campo de setembro. Já o ponto M50 teve sucessivos aumentos de vazão nos campos de novembro, março e junho, diminuindo sua vazão apenas no mês de setembro (quando ficou seco). Esse comportamento diferenciado indica que o fluxo que alimenta esses três pontos é mais lento, evidenciando, portanto, a importância do fluxo subsupercial e subterrâneo da água para o estudo de canais de pequena ordem, especialmente, para nascentes.

Os valores de TDS tendem ao aumento do mês de março para o mês de junho (Tabela 2), com exceção dos pontos M51, M60 e M62. Nos pontos perenes, o TDS tende a se comportar de duas maneiras: i) os valores 


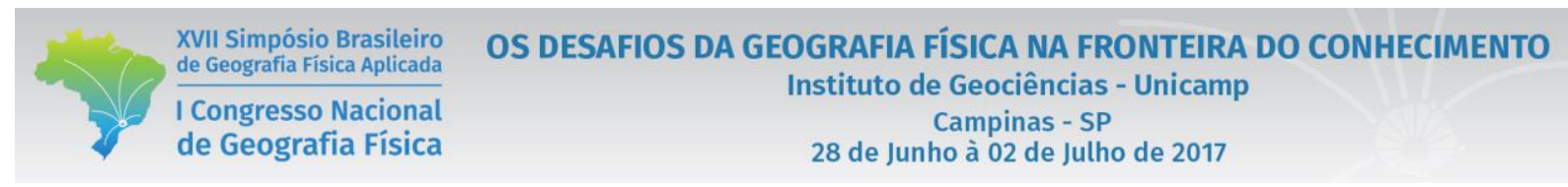

absolutos aumentam de novembro a junho e decaem no mês de setembro, como observado nos pontos M10, M22, M70, M71 e M92; ii) os valores absolutos decaem do mês de novembro para março, aumentam no mês de junho e voltam a decair no mês de setembro, conforme observado nos pontos M11, M12, M80 e M81.

Tabela 2: Resultados laboratoriais de sólidos totais dissolvidos e condutividade elétrica.

\begin{tabular}{|c|c|c|c|c|c|c|c|c|c|c|c|c|c|c|c|c|c|}
\hline \multirow[t]{2}{*}{$\begin{array}{l}\text { Micro- } \\
\text { bacia }\end{array}$} & \multicolumn{2}{|c|}{ Amostra } & \multicolumn{3}{|c|}{ TDS (mg/L) } & \multicolumn{4}{|c|}{$\mathrm{EC}(\mu \mathrm{S} / \mathrm{cm})$} & \multicolumn{4}{|c|}{ Alcalinidade (mg/L $\left.\mathrm{CaCO}_{3}\right)$} & \multicolumn{4}{|c|}{ Sílica $(\mathrm{mg} / \mathrm{L})$} \\
\hline & & Nov & Mar & Jun & Set & Nov & Mar & Jun & Set & Nov & Mar & Jun & Set & Nov & Mar & Jun & Set \\
\hline \multirow{7}{*}{1} & M10 & 30 & 35 & 45 & 18 & 17,54 & 12,54 & 15,04 & 15,59 & 5,5 & 6 & 12 & 8 & 5,17 & 3,80 & 8,38 & 16,17 \\
\hline & M11 & 50 & 40 & 112 & 46 & 26,01 & 16,68 & 21,35 & 16,00 & 9,5 & 8,5 & 11 & 8 & 5,63 & 4,72 & 12,50 & 15,25 \\
\hline & M12 & 40 & 20 & 61 & 23 & 23,04 & 15,76 & 19,40 & 23,56 & 9 & 9 & 11,5 & 8 & 5,17 & 4,72 & 2,88 & 16,17 \\
\hline & M20 & $\mathrm{S}$ & 26 & 45 & $*$ & $\mathrm{~S}$ & 10,71 & 10,71 & 14,21 & S & 5 & 6,5 & 8 & $\mathrm{~S}$ & 2,43 & 6,09 & 12,96 \\
\hline & M21 & $\mathrm{S}$ & 29 & 43 & S & $\mathrm{S}$ & 10,27 & 10,27 & S & $S$ & 6 & 8 & S & $\mathrm{S}$ & 2,88 & 8,84 & S \\
\hline & M22 & 40 & 41 & 45 & 28 & 30,70 & 16,02 & 23,36 & 19,98 & 15 & 9 & 7,5 & 11 & 6,55 & 4,72 & 9,75 & 19,83 \\
\hline & M31 & 70 & 49 & 89 & S & 40,74 & 25,44 & 33,09 & $\mathrm{~S}$ & 22 & 15 & 15,5 & S & 8,38 & 6,09 & 12,04 & $\mathrm{~S}$ \\
\hline \multirow{7}{*}{2} & M40 & $\mathrm{S}$ & 36 & 110 & $S$ & $\mathrm{~S}$ & 15,50 & 15,50 & S & $\mathrm{S}$ & 8 & 10 & S & $\mathrm{S}$ & 3,80 & 12,96 & $\mathrm{~S}$ \\
\hline & M41 & S & 29 & 61 & S & S & 16,83 & 16,83 & S & S & 8,5 & 12 & S & S & 2,88 & 9,30 & $\mathrm{~S}$ \\
\hline & M42 & S & 51 & 57 & S & S & 22,16 & 22,16 & S & S & 11 & 10 & S & S & 4,72 & 7,92 & $\mathrm{~S}$ \\
\hline & M50 & $S$ & 56 & 58 & $S$ & S & 29,12 & 29,12 & S & $S$ & 15,5 & 18 & $S$ & $S$ & 3,80 & 9,30 & $\mathrm{~S}$ \\
\hline & M51 & S & 43 & 42 & S & S & 22,10 & 22,10 & S & S & 11,5 & 11 & S & S & 3,34 & 7,46 & $\mathrm{~S}$ \\
\hline & M60 & S & 60 & 36 & S & S & 26,75 & 26,75 & S & S & 13 & 8 & S & S & 5,63 & 7,92 & $\mathrm{~S}$ \\
\hline & M61 & $\mathrm{S}$ & 56 & $\mathrm{~S}$ & $S$ & S & 21,72 & S & $S$ & $S$ & 10 & $\mathrm{~S}$ & $S$ & $\mathrm{~S}$ & 6,09 & $\mathrm{~S}$ & $\mathrm{~S}$ \\
\hline \multirow{7}{*}{3} & M71 & 20 & 29 & 31 & 22 & 20,58 & 11,81 & 16,20 & 12,61 & 7,5 & 6,5 & 8 & 7 & 3,80 & 2,88 & 4,26 & 9,75 \\
\hline & M72 & 20 & 23 & 32 & 33 & 23,75 & 14,79 & 19,27 & 15,29 & 9,5 & 8,5 & 8 & 8 & 5,17 & 3,80 & 4,72 & 12,50 \\
\hline & M80 & 30 & 10 & 32 & 23 & 16,00 & 9,42 & 12,71 & 11,18 & 5,5 & 5 & 8 & 4 & 3,80 & 2,88 & 3,34 & 9,75 \\
\hline & M81 & 20 & 9 & 39 & 22 & 21,04 & 11,54 & 16,29 & 10,82 & 8 & 6,5 & 12,5 & 4 & 3,34 & 2,88 & 4,26 & 9,75 \\
\hline & M90 & 10 & 9 & 16 & 17 & 15,30 & 15,76 & 15,53 & 12,10 & 7,5 & 9 & 9,5 & 8 & 3,34 & 2,88 & 1,97 & 11,13 \\
\hline & M91 & 20 & 10 & 43 & $*$ & 20,37 & 15,34 & 17,86 & 15,09 & 10 & 12 & 8,5 & 9 & 3,80 & 3,80 & 2,88 & 12,50 \\
\hline & M92 & 10 & 13 & 38 & 33 & 31,50 & 16,66 & 24,08 & 14,76 & 9,5 & 9 & 23,5 & 8 & 4,26 & 3,34 & 2,88 & 12,04 \\
\hline
\end{tabular}

Pode-se observar que de junho para setembro, os valores têm uma substancial queda, isto porque em um acumulado de dez dias do campo de setembro foram precipitados $30 \mathrm{~mm}$, enquanto no campo de junho, apenas 7,8mm. Outra especificidade encontrada, é que as nascentes perenes apresentaram valores absolutos de TDS menores que nascentes intermitentes.

De modo similar ao TDS, a EC apresentou dois padrões de comportamento, sendo eles: i) os valores absolutos apresentam sucessivas quedas em março e junho e aumentam em setembro, como em M10, M11, M12, M72, M80 e M91; ou ii) diminuem os valores absolutos de novembro para março, aumentam em junho e voltam a diminuir em setembro, como em M22, M70, M71 e M92.

De maneira geral, os valores tendem a diminuir de novembro para março, visto que há uma grande concentração de precipitação (aumentando, portanto, a quantidade de solvente). A queda dos valores de março a junho, observada em um considerável número de pontos, está atrelada a queda nos valores de acumulado de precipitação, condicionando então, a uma maior concentração de íons. 


\section{OS DESAFIOS DA GEOGRAFIA FÍSICA NA FRONTEIRA DO CONHECIMENTO \\ Instituto de Geociências - Unicamp \\ Campinas - SP \\ 28 de Junho à 02 de Julho de 2017}

A EC não apresenta um comportamento padrão em função da hierarquia fluvial. Os pares de pontos nascentes/canal de primeira ordem apresentam dois padrões: i) tem a ter um aumento da EC, como em M10/M11, M40/M41, M80/M81, M90/M91; ii) tendem a diminuir, como em M20/M21, M50/51, M60/M61, M70/M71. Os três pontos de análise dos canais de segunda ordem e segunda magnitude, apresentaram comportamentos distintos: i) O ponto M12, que recebe água dos pontos M11 e M21, apresenta valores de EC intermediários entre esses pontos, ou seja, a mistura da água desses dois canais ocorre de maneira equilibrada; ii) O ponto M42, formado pela confluência dos pontos M41 e M51, apresenta EC bem próxima ao do ponto M51; iii) O ponto M92, formado pelos pontos M81 e M91, apresenta EC maior do que a de seus formadores, podendo estar relacionada a contribuição do aquífero ou a decomposição de matéria orgânica no canal.

Ao analisarmos os pontos de segunda ordem e terceira magnitude, a realidade de cada microbacia não é muito alterada, visto que a junção dos pontos M12 e M31, originam M22 com EC também intermediaria. O ponto M62 apresenta EC menor que M42 e M61, e o ponto M72, apresenta valores maiores que os pontos M71 e M92.

De maneira geral, a microbacia 2 apresenta os maiores valores de EC, o que é esperado, visto que esta é a bacia com menor vazão. A microbacia 1, apresenta valores intermediários de vazão e EC. Já a microbacia 3 apresenta os menores valores de EC, condizentes com o maior volume de água entre as microbacias. Já o TDS não apresentou correlação clara com os parâmetros EC e vazão.

Os valores de sílica, de maneira geral, diminuem de novembro para março, aumentam em junho e setembro. O mês de setembro (final do período seco e mínimo hidrológico) é quando a sílica apresenta os valores mais elevados do rol. Os pontos M12, M90 e M92 se comportam de maneira distinta dos demais, visto que seus valores diminuem de novembro até junho, tendo aumento apenas em setembro. Com relação a influência da hierarquia fluvial, a maioria dos pontos tende, ou a manter, ou aumentar, o valor de sílica no sistema. A microbacia 1 apresenta os maiores valores de sílica, seguida pela microbacia 2 e pela microbacia 3.

A alcalinidade, de maneira geral, tende a diminuir seu valor de novembro para março, aumentar em junho e diminuir novamente em setembro. Os valores mais elevados estão concentrados no mês de junho (auge do período seco). Ademais, pode-se observar que a alcalinidade tende a sofrer aumentos com o aumento da hierarquia fluvial, o que só não é observado nos pares de pontos M50/M51 e M60/M61. Os valores de alcalinidade são maiores na microbacia 2, intermediários na microbacia $1 \mathrm{e}$ tem os menores valores na microbacia 3. 


\section{OS DESAFIOS DA GEOGRAFIA FÍSICA NA FRONTEIRA DO CONHECIMENTO \\ Instituto de Geociências - Unicamp \\ Campinas - SP \\ 28 de Junho à 02 de Julho de 2017}

\section{Considerações Finais}

Não há diferenciações significativas entre as microbacias quanto à hidrogeoquímica, já que a área não apresenta uma grande variação no embasamento geológico, mas há significativas diferenciações com a vazão, que por sua vez, é condicionada por formas e processos geomorfológicos;

Os máximos de concentração dos parâmetros analisados não coincidem. Seria interessante avaliar ponto a ponto, mas há, também, uma resposta para a diferença de velocidade na mobilização (lixiviação) de cada composto;

A EC não está diretamente relacionada ao TDS em alguns casos. Alterações na concentração de sílica, que é quimicamente inerte, alteram o valor de TDS e não alteram a EC. Isto demonstra a importância da escolha do método para a determinação do TDS;

É pertinente que em futuros estudos sejam analisadas o papel dos fluxos higroscópicos, visto que por terem menor velocidade, ficam mais tempo em contato com a rocha e/ou com as coberturas superficiais de alteração, podendo alterar o tipo e a concentração de compostos químicos na água.

\section{Agradecimentos}

Ao CNPq pelo financiamento da pesquisa; ao Laboratório de Geomorfologia do IGC/UFMG; ao grupo de pesquisa Geomorfologia e Recursos Hídricos (UFMG-UFJF).

\section{Bibliografia}

ALBARÈDE, F. Geoquímica: uma introdução. São Paulo: Oficina de Textos, 2011.

APHA - AMERICAN PUBLIC HEALTH ASSOCIATION; AWWA - AMERICAN WATER WORKS ASSOCIATION; WEF - WATER ENVIRONMENT FEDERATION. Standard Methods for Examination of Water and Wastewater. Washington: APHA, 22 har., Cdr Edition, 2012.

BENDA, L. HASSAN, M. A., CHURCH, M., \& MAY, C. L. Geomorphology of Steepland Headwaters: The Transition From Hillslopes To Channels1. Journal of the American Water Resources Association, v. 41, n. 4, 2005. $835 \mathrm{p}$.

BORGA, M. STOFFEL, M., MARCHI, L., MARRA, F., JAKOB, M.. Hydrogeomorphic response to extreme rainfall in headwater systems: flash floods and debris flows. Journal of Hydrology, v. 518, p. 194-205, 2014.

CRUZ, L. A. Áreas Verdes e Espaço Urbano: A mata do Krambeck e a cidade de Juiz de Fora em Minas Gerais. 2016. 112 f. Dissertação (Mestrado em Ecologia)- Instituto de Ciências Biológicas, Universidade Federal de Juiz de Fora, Juiz de Fora, 2016.

DAVIS, S. N. Hydrogeology. New York : J. Willey, 1966.

DUNNE, T. Formation and controls of channel networks. Prog. Phys. Geogr., v. 4, p. 211-239, 1980.

FARIA, A. P. Dinâmica e Fragilidade das Bacias Fluviais de Primeira Ordem. 1996. 216 f. Tese (Doutorado em Ciências)- Universidade Federal do Rio de Janeiro, Rio de Janeiro, 1996. 
FEITOSA, A. C.; MANOEL-FILHO, J. Hidrogeologia: conceitos e aplicações. Fortaleza: CPRM, LABHID-UFPE, 2000.

FELIPPE, M. F.; MAGALHÃES JR, A. P. A contribuição das nascentes na desnudação geoquímica: borda oeste da Serra do Espinhaço Meridional. Revista Brasileira de Geomorfologia, v. 17, p. 79-92, 2016.

Análise da variabilidade da vazão das nascentes no Parque das Mangabeiras (Belo Horizonte-MG) em relação aos seus condicionantes ambientais. In: XIII Simpósio Brasileiro de Geografia Física Aplicada, 2009, Viçosa-MG. Anais .... Viçosa: UFV, 2009.

Desenvolvimento de uma tipologia hidrogeomorfológica de nascentes baseada em estatística nebulosa multivariada. Revista Brasileira de Geomorfologia, v. 15, p. 393-409, 2014.

FONSECA, C. R. Composição, Estrutura e Diversidade da Comunidade Arbórea de um Fragmento Urbano de Floresta Estacional Semidecidual (Juiz de Fora, MG, Brasil). 2012. 52 f. Dissertação (Mestrado em Ecologia)Instituto de Ciências Biológicas, Universidade Federal de Juiz de Fora, Juiz de Fora, 2012.

GOMI, T.; SIDLE, R. C.; RICHARDSON, J. S. Understanding processes and downstream linkages of headwater systems: headwaters differ from downstream reaches by their close coupling to hillslope processes, more temporal and spatial variation, and their need for different means of protection from land use. BioScience, v. 52, n. 10, p. $905-$ 916, 2002.

HAIGH, M. Environmental change in headwater peat wetlands, UK. In: KRECEK, J.; HAIGH, M. (Org.). Environmental Role of Wetlands in Headwaters, p. 237-255, 2006.

IBGE - Instituto Brasileiro de Geografia e Estatística. Carta Topográfica de Juiz de Fora. ed. 1996. SF-23-X-DIV-1. Escala: 1:50.000.

Mapa de Unidades do Relevo do Brasil. 2 ed. 2006. Escala: 1:5.000.000.

LEVISON, J., LAROCQUE, M., FOURNIER, V., GAGNÉ, S., PELLERIN, S., OUELLET, M. A. Dynamics of a headwater system and peatland under current conditions and with climate change. Hydrological Processes, v. 28, n. 17, p. 4808-4822, 2014.

MARQUES NETO, R.; PEREZ FILHO, A. ; OLIVEIRA, T. A. . Itatiaia Massif: morphogenesis of southeastern brazilian highlands. In: VIEIRA, B. C.; SALGADO, A. A. R.; SANTOS, L. J. C.. (Org.). Landscapes and Landforms of Brazil. Dordrecht: Springer, 2015, p. 299-308.

MONTGOMERY, D. R.; DIETRICH, W. E. Source areas, drainage density, and channel initiation. Water Resources Research, v. 25, n. 8, p. 1907-1918, ago, 1989.

Where do channels begin? Nature, v. 336, n. 6196, p. 232-234, nov 1988.

NOCE, C. M.; PEDROSA-SOARES, A. C.; SILVA, L. C.; ALKMIM, F. F. O Embasamento Arqueano e Paleoproterozóico do Orógeno Araçuaí. Geonomos, v. 15, n. 1, p. 17-23, 2007.

OLIVEIRA, A. Fragilidade Ambiental no Setor Norte do Município de Juiz de Fora (MG): subsídios da geomorfologia ao planejamento urbano. 2016. 148 f. Dissertação (Mestrado em Geografia)- Instituto de Ciências Humanas, Universidade Federal de Juiz de Fora, Juiz de Fora, 2016.

OLIVEIRA, D. E.; ASSIS, D. C.; PIMENTEL, F. O.; FERREIRA, C. C. M. Considerações Sobre a Precipitação na Bacia Hidrográfica do Rio Paraibuna. In: Congresso Brasileiro de Geógrafos, 7. Anais... Vitória, 2014.

PALMER, M.; HOPE, D.; BILLETT, M. F.; DAWSON, J. J.; BRYANT, C. L. Sources of organic and inorganic carbon in a headwater stream: evidence from carbon isotope studies. Biogeochemistry, v. 52, n. 3, p. 321-338, 2001.

ROCHA, G. C. Riscos Ambientais: análise e mapeamento em Minas Gerais. Juiz de Fora : UFJF, 2005.

SANTIAGO, B. S.; REZENDE, R. F.; FERREIRA, C. C. M. Reserva Biológica Municipal de Poço D’Anta, Juiz de Fora/MG- aspectos da fragmentação de habitat e efeito de borda. Gaia Scientia, v. 1, n. 1, p. 53-66, 2007.

SCHUMM, S. A. Evolution of drainage systems and slopes in badlands at Perth Amboy, New Jersey. Geological society of America bulletin, v. 67, n. 5, p. 597-646, 1956. 


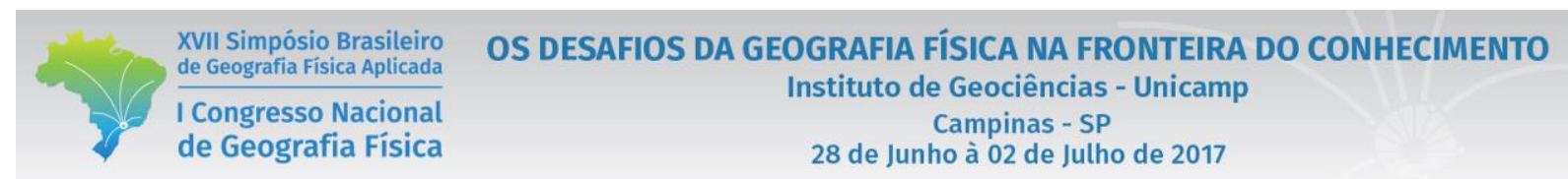

STRAHLER, A. N. Quantitative analysis of watershed geomorphology. Eos, Transactions American Geophysical Union, v. 38, n. 6, p. 913-920, 1957.

SAADI A. Ensaio sobre a morfotectônica de Minas Gerais: tensões intraplaca, descontinuidades crustais e morfogênese. Tese (Professor Titular), Instituto de Geociências, Universidade Federal de Minas Gerais, 1991

RICCOMINI C. O rift continental do sudeste do Brasil. Tese (doutorado), Instituto de Geociências, Universidade de São Paulo, 1989. 